\title{
Direct Analysis of Multiphase Switched-Capacitor Networks Using Signal-Flow Graphs
}

\author{
ADAM DA̧BROWSKI, MEMBER, IEEE, AND GEORGE S. MOSCHYTZ, FELLOW, IEEE
}

\begin{abstract}
A by-inspection analysis method for multiphase switchedcapacitor (SC) networks using signal-flow graph (SFG) techniques is presented. The method is quite general; no restrictions on the networks are required. It is also very simple and direct in the sense that it allows an immediate, by-inspection derivation of the SFG merely on the basis of a given SC network. The method is primarily useful for the hand analysis and design of small and medium-size SC networks (e.g., building blocks of modular SC systems). It may also serve as a tool for the symbolic computer analysis and for the synthesis of general multiphase SC networks. Illustrative examples demonstrate the simplicity and efficiency of the method.
\end{abstract}

\section{INTRODUCTION}

$\mathrm{S}$ IGNAL-flow graph (SFG) techniques have proven to be very useful for the design and hand analysis of small and medium-size switched-capacitor (SC) networks, e.g., of SC modules and building blocks of larger systems [1]-[3]. Although many different methods for the derivation of SFG's for SC networks have already been proposed (e.g., [1]-[10]), they are still relatively complicated and time consuming. Many of them are also subject to restrictions on the class of networks that can be analyzed. Furthermore, most of the methods are not direct in the sense that they require some intermediate steps comprising auxiliary circuit transformations [4], or even algebraic or topological manipulations [5]-[10]. The direct methods are limited to the analysis of biphase [2], [3] or strayinsensitive SC networks [1], [2].

The SFG analysis method described in this paper is a generalization of the direct method introduced in [2] and an extension of [1]. The latter is restricted to a special class of networks (the so-called SSN-type networks) comprising only source and sink nodes as explained further on. ${ }^{1}$ Since new technologies (such as GaAs) relax the restriction of parasitic insensitivity (while admittedly imposing others) [11]-[13], not only multiphase but also general SC circuits with, say, unity-gain buffer amplifiers

Manuscript received February 27, 1989; revised August 31, 1989. This paper was recommended by Associate Editor J. Mavor.

A. Dabrowski is with the Institute of Electronics and Telecommunications, Department of Electrical Engineering, Technical University of Poznań, PL-60 965 Poznań, Poland.

G. S. Moschytz is with the Institute of Signal and Information Processing, Department of Electrical Engineering, Swiss Federal Institute of Technology, ETH Centre, CH-8092, Zurich, Switzerland.

IELE Log Number 8934172

${ }^{1}$ Note that all stray insensitive circuits are contained in this class [2].

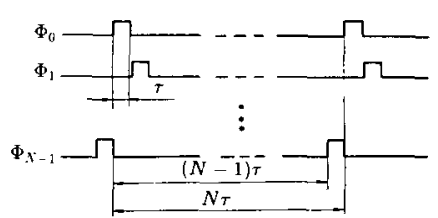

Fig. 1. A symmetric $N$-phase clock.

instead of conventional op amps may be expected in the near future. This was the motivation for extending the SFG analysis method beyond SSN-type networks so as to cover the most general multiphase SC networks. Since the treatment of multiphase SSN-type SC networks was only summarized in [1], the detailed rationale behind the byinspection analysis technique is first presented here. The analysis of general, nonrestricted networks then follows, along with some illustrative examples.

\section{ANalysis of SSN-Type SC Networks}

We consider multiphase SC networks driven by a symmetrical $N$-phase clock $^{2}$ as shown in Fig. 1. In this section we assume furthermore that the SC networks under consideration contain only three types of nodes (except for the grounded reference node):

i) nodes driven by ideal voltage sources (e.g., op amp outputs), the source nodes;

ii) nodes at virtual ground, the sink nodes;

iii) nodes connecting capacitors with switches; these nodes do not appear in the SFG and need not be considered further because they are also connected either to source or to sink nodes or are disconnected from the network (when corresponding switches are open).

We refer to SC networks belonging to the class defined above as source-sink-node-type (SSN) SC networks. ${ }^{3}$ All possible switching transitions for the capacitor plate of such a network are illustrated in Fig. 2. Switch connec-

\footnotetext{
${ }^{2}$ Generalization of our considerations to an arbitrary (i.e., nonsymmetric) $N$-phase clock is straightforward but not relevant to the analysis described here.

${ }^{3}$ Note that the definition of SSN-type networks can be applied to any active network; SSN-type networks are those containing source and sink nodes only.
} 


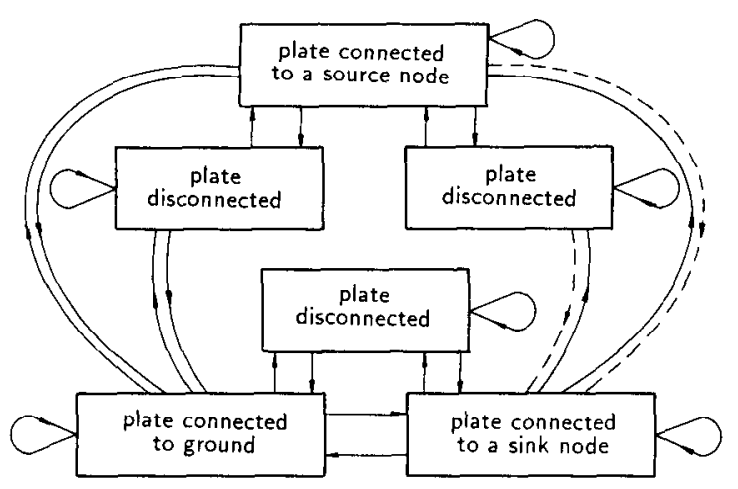

Fig. 2. Switching possibilities for any capacitor plate of a multiphase SSN-type SC network.

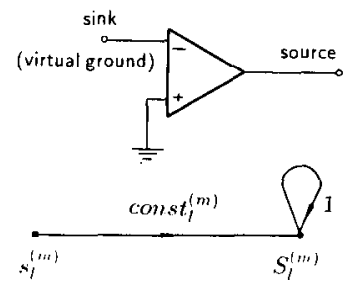

(a)

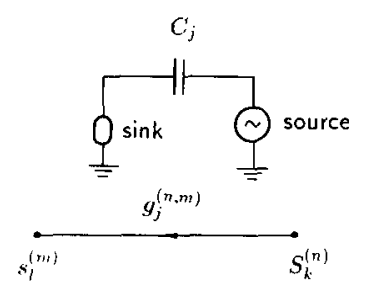

(b)
Fig. 3. SFG subgraphs (a) Subgraph modeling $l$ th op amp in $m$ th switching phase. (b) Branch modeling capacitor $C_{i}$ switched between $k$ th source node in $n$th phase and $l$ th sink node in $m$ th phase.

tions designated by dashed lines are not allowed for stray-insensitive SC networks [14]. Hence, the class of SSN-type SC networks is slightly broader than that of stray-insensitive networks.

In the following considerations we shall assume that the only active elements contained in an SSN-type network are inverting op amps. This is a minor restriction because in the general case (considered in Section III-3.3) an SSN-type network can also contain finite-gain amplifiers controlled by op amp outputs. The op amp outputs are, therefore, called independent source nodes and the finite-gain amplifier outputs dependent source nodes. Note that an independent source node can be represented by a grounded norator, and a sink node by a grounded nullator [20].

It can be shown that the SFG of an SSN-type SC network containing only inverting op amps is composed of two types of subgraphs, namely:

i) subgraphs modeling op amps (Fig. 3(a)), and

ii) branches modeling switched capacitors (Fig. 3(b)).

The SFG will contain only source and sink nodes; ${ }^{4}$ these correspond to the source and sink nodes of the SC network. Each source and each sink node of the SC network generates at most $N$ nodes in the SFG, and each op amp at most $N$ subgraphs of the type in Fig. 3(a). These correspond to the individual switching phases $n=$ $0,1, \cdots, N-1$. If for any switching phase, e.g., for the phase $m$, the inverting input and the output of the $l$ th op

\footnotetext{
${ }^{4}$ We shall show later that all SFG sink nodes may be eliminated by
}

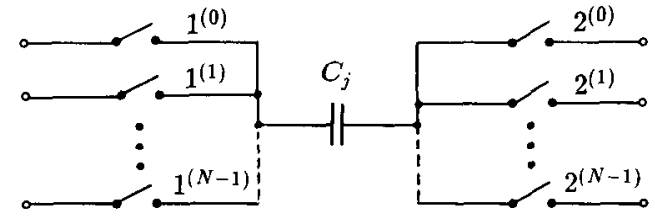

Fig. 4. A multiphase SC $C_{j}$.

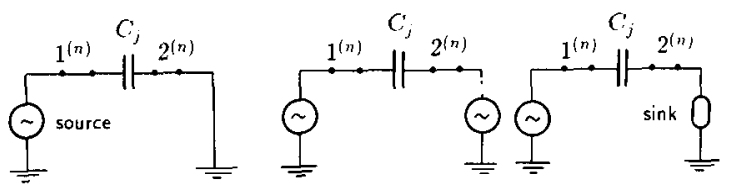

(a)

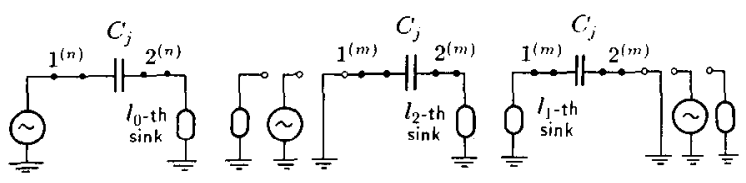

(b)

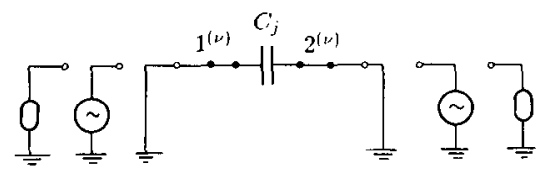

(c)

Fig. 5. Particular switching situations for a capacitor $C_{j}$. (a) Charge. (b) Charge transfer. (c) Discharge.

amp are short circuited by a switch, then the nodes $S_{l}^{(m)}$ (source node) and $s_{l}^{(m)}$ (sink node), and the corresponding subgraph of the type in Fig. 3(a) do not appear in the SFG.

Note that op amp subgraphs (Fig. 3(a)) always start at sink nodes and end in source nodes, whereas the capacitor branches start at source nodes and end in sink nodes. The transmittance designated in Fig. 3(a) as const ${ }_{l}^{(m)}$ is arbitrary but not equal to zero. Therefore, choosing the simplest and most convenient possibility, we let const ${ }_{l}^{(m)}$ $=1$ for all $l$ and $m$. With this assumption the only parameters appearing in the SFG are the transmittances $g_{j}^{(n, m)}$ where $n$ is the switching phase at the source node and $m$ the switching phase at the sink node (Fig. 3(b)). We shall now show that these transmittances can be determined directly by inspection of the SC network. To this end we consider a general multiphase switched capacitor $C_{j}$ shown in Fig. 4. Switches $1^{(n)}$ and $2^{(n)}, n=$ $0,1, \cdots, N-1$, are closed in the $n$th phase, provided they are activated. Switches that are not activated remain open in our model and are omitted in the actual circuit.

The following observations can now be made.

i) A capacitor is charged in the $n$th phase if one of its plates is connected to a source node and the other, directly or indirectly, to ground (via a source node or a sink node), see Fig. 5(a).

ii) The charge of a capacitor (acquired during the $n$th

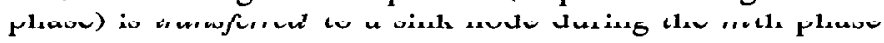




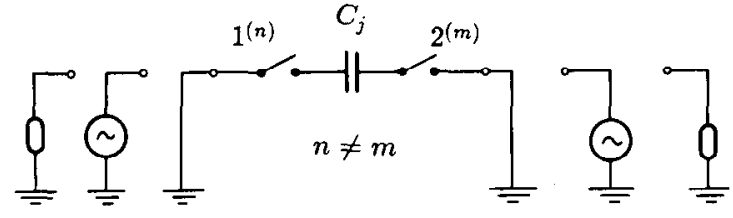

Fig. 6. Examples of meaningless circuit configurations.

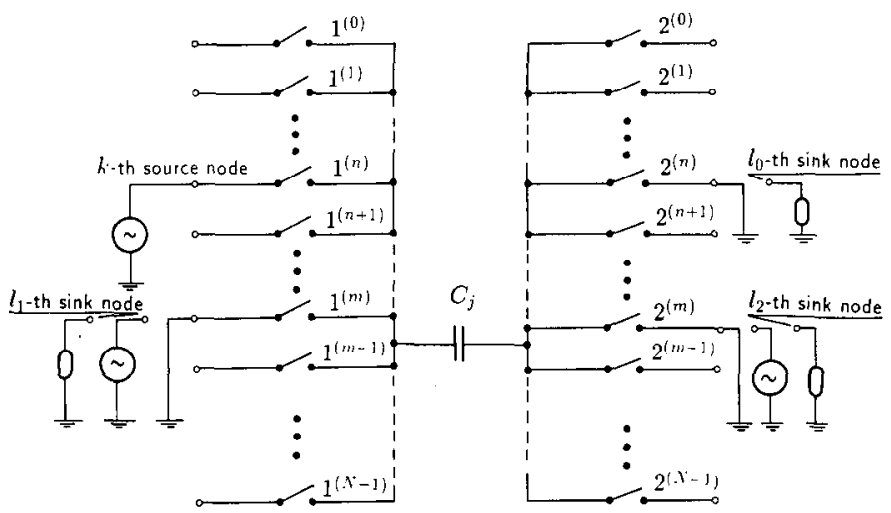

Fig. 7. A multiphase switched capacitor $C_{j}$ of an SSN-type SC network.

("transfer charge") if at least one capacitor plate is connected to a sink node and the other-directly or indirectly-to ground during the $m$ th phase (Fig. 5(b)). Note that in general $m=(n+\mu) \bmod N, \mu=0,1, \cdots$, $N-1$.

iii) A capacitor is discharged during any phase, say during the phase $\nu, \nu=0,1, \cdots, N-1$, if both capacitor plates are shorted to ground, directly or indirectly, during that phase (Fig. 5(c)).

Note that the conditions for capacitor charge, charge transfer, and discharge are not mutually exclusive; they can take place simultaneously during the same phase.

The following observations are direct consequences of those above.

iv) In order for a capacitor to influence the surrounding network (be charged, or discharged with a transfer charge) in the $n$th phase, both capacitor plates must be connected directly, or indirectly (via a source node or a sink node), to ground in that phase. Thus no charge or discharge of capacitor $C_{j}$ can take place in the configuration of Fig. 6.

v) Each capacitor of an SSN-type SC network must have at least one source node and one sink node connected to it; without at least one source node at some phase period $n \tau$, the capacitor will never be charged; without at least one sink node at some phase period $m \tau$, the capacitor will never transfer its charge to the surrounding network.

vi) A capacitor charged in the $n$th phase, say by the $k$ th source node connected during this phase to the left capacitor plate (Fig. 7), can transfer its charge in an SSN-type SC network through at most three sink nodes, designated in Fig. 7 as nodes $l_{0}, l_{1}$, and $l_{2}$; the $l_{0}$ th node is that connected to the right capacitor plate during the

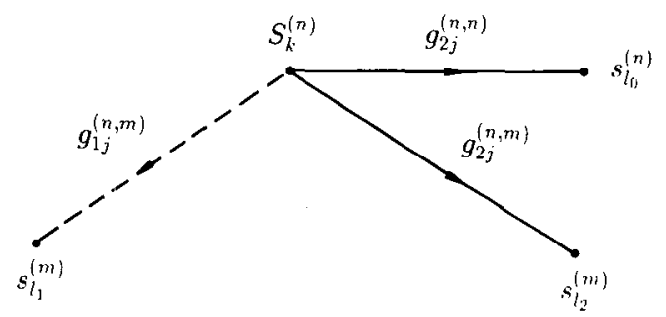

Fig. 8. Branches possibly generated in the SFG of an SSN-type SC network by a capacitor $C_{j}$ charged in the $n$th phase.

$n$th phase, and the $l_{1}$ th node and the $l_{2}$ th node are connected to the left and to the right capacitor plate, respectively, during the $m$ th phase, $m$ being any phase within the following time period $T=N \tau$. The charge transfer in the $m$ th phase takes place only if there is no phase $\nu$ in between phases $n$ and $m$ in which the capacitor is discharged.

The following proposition follows directly from observation vi).

Proposition 1: In the SFG of a multiphase SSN-type SC network, at most three branches, corresponding to a particular capacitor, can leave a source node (Fig. 8).

Note that the transmittance $g_{1}^{(n, m)}$ of the branch, drawn in Fig. 8 by a dashed line, results from switching the left plate of the capacitor $C_{j}$ between a source node and a sink node. This is, however, not allowed for stray-insensitive networks (cf., Fig. 2). Thus we can formulate our second proposition.

Proposition 2: In the SFG of a multiphase stray-insensitive SC network, at most two branches, corresponding to a particular capacitor, can leave a source node.

Finally the values of the transmittances $g_{2_{j}}^{(n, n)}, g_{2_{j}}^{(n, m)}$, and $g_{1_{j}}^{(n, m)}$ are determined by the following proposition and are summarized in Table I. They follow directly from observations i)-vi). (Note that $\tau$ is the clock subperiod; it will later be normalized to unity.)

Proposition 3: Assume, as in Fig. 7, that node $1^{(n)}$ connects a capacitor $C_{j}$ to a $k$ th source node (then all other nodes in Fig. 7 are potential sink nodes).

First, assume that switch $2^{(n)}$ connects the capacitor $C_{j}$ to a sink node during the $n$th phase. If within the following time period $N-\tau$ there exists a phase $\nu$ such that both switches $1^{(\nu)}$ and $2^{(\nu)}$ are closed, thereby discharging $C_{j}$ in phase $\nu$, then

$$
g_{2 j}^{(n, n)}=\frac{C_{j}}{\tau}
$$

otherwise, i.e., if no such discharging in any phase $\nu$ occurs, then

$$
g_{2 j}^{(n, n)}=\frac{C_{j}}{\tau}\left(1-z^{-N}\right) .
$$

Second, assume that switch $2^{(m)}$ connects a capacitor $C_{j}$ to a sink node during a phase $m \neq n, m=n+\mu$ (or 
TABLE I

Transmittances Resulting from the Capacitor $C_{j}$ Connected TO THE SOURCE NODE BY THE SWITCh $1^{(n)}$

\begin{tabular}{|c|c|c|c|c|c|c|}
\hline \multicolumn{3}{|c|}{ Switching configurations } & \multicolumn{4}{|c|}{ Transmittance values } \\
\hline \multirow{4}{*}{$\begin{array}{l}\text { Switch } \\
2^{(n)}\end{array}$} & \multirow{4}{*}{$\begin{array}{l}\text { Switches } \\
1^{(\nu)} \text { and } 2^{(\nu)} \\
\nu \text { is a discharging } \\
\text { phase inbetween } \\
\text { phases } n \text { and } m\end{array}$} & \multirow{4}{*}{$\begin{array}{c}\text { Switches } \\
1^{(m)} \text { and } 2^{(m)} \\
m \text { is a transfer } \\
\text { charge phase } \\
m=(n+u) \bmod N\end{array}$} & \multicolumn{4}{|c|}{ Arbitrary SC network's } \\
\hline & & & \multicolumn{3}{|c|}{ SSN-type SC networks } & \multirow[b]{3}{*}{$g_{1}^{(n, n)}$} \\
\hline & & & \multicolumn{2}{|c|}{$\begin{array}{c}\text { Stray-insensitive } \\
\text { SC networks }\end{array}$} & \multirow[b]{2}{*}{$g_{1 ;}^{(n, m)}$} & \\
\hline & & & $g_{2 j}^{(n, n)}$ & $g_{2 j}^{\left(n_{1}, n_{2}\right)}$ & & \\
\hline open & arbitrary & arbitrary & 0 & 0 & 0 & 0 \\
\hline \multirow{2}{*}{$\begin{array}{l}\text { closed to } \\
\text { A sink node }\end{array}$} & both closed & arbitrary & $C_{j}$ & \multirow{6}{*}{ - } & \multirow{6}{*}{-} & $-C_{j}$ \\
\hline & at least one open & st least one open & $C_{j}\left(1-z^{-N}\right)$ & & & $-C_{j}\left(1-z^{-K}\right)$ \\
\hline \multirow{2}{*}{$\begin{array}{l}\text { dosed to } \\
\text { ground }\end{array}$} & both cloned & atitrary & 0 & & & $-C_{j}$ \\
\hline & At lesst one open & At least one open & 0 & & & $-C_{j}\left(1-z^{-N}\right)$ \\
\hline \multirow{2}{*}{$\begin{array}{c}\text { closed to } \\
\text { a source node }\end{array}$} & boch cloned & arbitrany & 0 & & & $-\bar{C}_{j}$ \\
\hline & at least onc open & at least one open & 0 & & & $-C_{i}\left(1-z^{-x}\right)$ \\
\hline \multirow{5}{*}{ rloked } & closed & Arbitrary & \multirow{5}{*}{-} & 0 & 0 & \multirow{5}{*}{ 工 } \\
\hline & \multirow{4}{*}{$\begin{array}{l}\text { At least } \\
\text { ane open }\end{array}$} & $\begin{array}{l}1^{(m)} \text { closed to ground } \\
\text { or to a source node } \\
2^{(m)} \text { closed to } \\
\text { a sink rode }\end{array}$ & & $-C_{j} z^{-\mu}$ & 0 & \\
\hline & & $\begin{array}{l}1^{(m)} \text { cloosed is } \\
\text { a tink node }\end{array}$ & & & & \\
\hline & & $\begin{array}{l}2^{(m)} \text { closed to ground } \\
\text { or to a source node }\end{array}$ & & 0 & $C_{j} z^{-\mu}$ & \\
\hline & & $\begin{array}{l}\text { both switches closed } \\
\text { to sink nodet }\end{array}$ & & $-\overline{C_{j}} z^{-\bar{H}}$ & $\overline{C_{j} z^{-4}}$ & \\
\hline
\end{tabular}

generally $m=(n+\mu) \bmod N, \mu=1,2, \cdots, N-1)$. If switches $2^{(n)}$ and $1^{(m)}$ are both closed in their respective phases, and if in no phase $\nu$ in between phases $n$ and $m$ are switches $1^{(\nu)}$ and $2^{(\nu)}$ both simultaneously closed, ${ }^{5}$ then

$$
g_{2 j}^{(n, m)}=-\frac{C_{j}}{\tau} z^{-\mu}
$$

otherwise, i.e., if at least one of the switches $2^{(n)}$ or $1^{(m)}$ is not closed, or if there exists a phase $\nu$ between phases $n$ and $m$ such that the switches $1^{(v)}$ and $2^{(\nu)}$ are both closed (thereby discharging $C_{j}$ during the $\nu$ th phase, i.e., before the $m$ th phase), then

$$
g_{2_{j}}^{(n, m)}=0
$$

Finally, assume that switch $1^{(m)}$ connects a capacitor $C_{j}$ to a sink node during a phase $m \neq n, m=(n+\mu) \bmod N$, $\mu=1,2, \cdots, N-1$. If switches $2^{(n)}$ and $2^{(m)}$ are both closed in their respective phases, and if in no phase $\nu$ in between phases $n$ and $m$ are switches $1^{(\nu)}$ and $2^{(\nu)}$ both simultaneously closed, then

$$
g_{1 j}^{(n, m)}=\frac{C_{j}}{\tau} z^{-\mu}
$$

otherwise, i.e., if at least one of the switches $2^{(n)}$ or $2^{(m)}$ is not closed, or if there exists a phase $\nu$ between phases $n$ and $m$ such that the switches $1^{(\nu)}$ and $2^{(\nu)}$ are both closed (thereby discharging $C_{j}$ in phase $\nu$ ), then

$$
g_{1 j}^{(n, m)}=0 \text {. }
$$

With the propositions above, the steps required for the derivation of the SFG of an $N$-phase SSN-type SC network can be summarized as follows.

i) Number all source and sink nodes of the SC network and check whether it belongs to the class of SSN-type

${ }_{1}^{5}$ Note
$L$ thaut, in general, $\nu=(n+1) \bmod N,(n+2) \bmod N, \cdots,(m-$

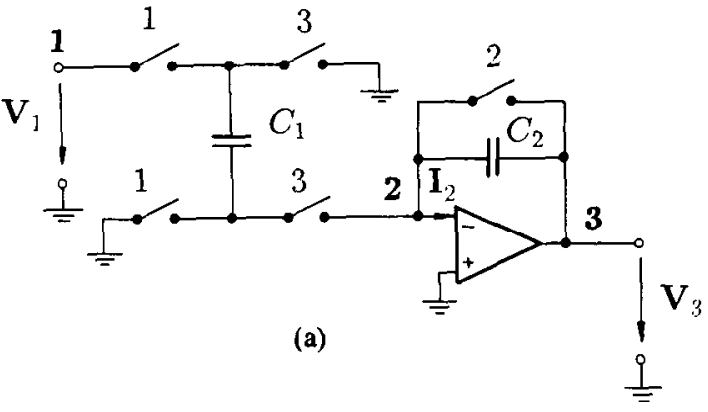

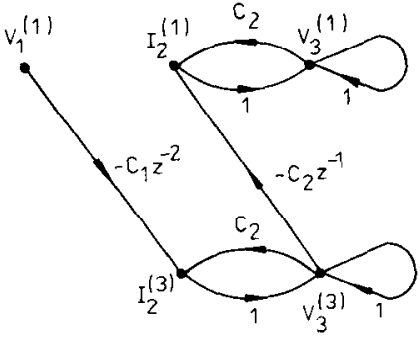

(b)

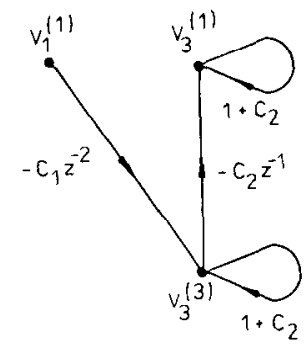

(c)
Fig. 9. 3-phase SC delay element [16]. (a) SC circuit. (b) Corresponding SFG. (c) Simplified SFG.

networks (i.e., every node must be either a source or a sink node).

ii) Draw all SFG nodes. First, $N$ SFG nodes for each network node may be drawn. Then, the nodes disconnected from the network in individual switching phases must be omitted from the SFG. Furthermore, those source-sink-node pairs corresponding to op amps with the inverting inputs shorted to their outputs in individual phases, must also be omitted.

iii) Connect the SFG nodes using op amp subgraphs of the type shown in Fig. 3(a) and switched capacitor branches of the type shown in Fig. 3(b). Transmittances of the latter are determined on the basis of Table I or, equivalently, by (1)-(6) of proposition 3 .

iv) Finally, simplify the SFG by eliminating all sink nodes by tying them to the corresponding source nodes. ${ }^{6}$ Thus for example, the node $s_{l}^{(m)}$ of a capacitor $C_{j}$ branch (Fig. 3(b)) can be directly connected to the node $S_{l}^{(m)}$ of the corresponding op amp subgraph (Fig. 3(a)). With a little practice, this simplified SFG (which corresponds to a Coates graph) can also be obtained directly by inspection.

The following example illustrates the above proccdure.

Example 1: Consider the 3-phase delay element of Fig. 9(a). This circuit was proposed in [16]. It contains two source nodes (nodes 1 and $\mathbf{3}$ in Fig. 9(a)) and one sink node (node 2 ). Because it is customary in the literature, we number the switching phases $1,2,3$ (and not $0,1,2$ as

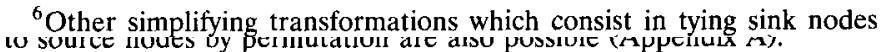


was done, for convenience, in the above theoretical considerations). In the SFG (Fig. 9(b)), the op amp is represented by two subgraphs of the type in Fig. 3(a). They connect nodes $I_{2}^{(1)}$ and $I_{2}^{(3)}$ with nodes $V_{3}^{(1)}, V_{3}^{(3)}$, respectively. Node 1 is represented by only one node with the signal $V_{1}^{(1)}$. This is because the node $\mathbf{1}$ is disconnected from the circuit in phases 2 and 3 , and therefore, the signals $V_{1}^{(2)}$ and $V_{1}^{(3)}$ are omitted from the SFG. On the other hand, each of the nodes 2 and 3 generates two nodes in the SFG, nodes $I_{2}^{(1)}, I_{2}^{(3)}$ and nodes $V_{3}^{(1)}, V_{3}^{(3)}$, respectively. Nodes 2 and 3 are not represented in the SFG in phase 2 because they are short circuited by a switch in this phase; in other words, $V_{3}^{(2)}=0$ and $I_{2}^{(2)}$ is arbitrary, and therefore, these signals are omitted. Capacitor $C_{1}$ generates an SFG branch from node $V_{1}^{(1)}$ to node $I_{2}^{(3)}$ since $C_{1}$ is connected (i.e., charged) to the source node 1 in phase 1 and to the sink node 2 (i.e., discharged) in phase 3 . The corresponding transmittance $-C_{1} z^{-2}$ is given by (3) with $n=1, m=3$, and $\mu=3-1=2 .{ }^{7}$ The SFG also contains three branches representing capacitor $C_{2}$. Two of them, namely those from nodes $V_{3}^{(1)}$ and $V_{3}^{(3)}$ to nodes $I_{2}^{(1)}$ and $I_{2}^{(3)}$, respectively, have transmittances equal to $C_{2}$ (according to (1)). This is due to the fact that the capacitor $C_{2}$ is short circuited (discharged) in phase 2 . The third branch, which also relates to capacitor $C_{2}$, starts in node $V_{3}^{(3)}$ and ends in node $I_{2}^{(1)}$. Its transmittance $-C_{2} z^{-1}$ follows from (3) for $n=3, m=1$, and $\mu=(1-$ $3) \bmod 3=1$. Capacitor $C_{2}$ generates no branch from phase 1 to phase 3 (i.e., from $V_{3}^{(1)}$ to $I_{2}^{(3)}$ ) because between these phases, i.e., in phase $2, C_{2}$ is discharged. Finally, tying the sink nodes, which represent the signals $I_{2}^{(1)}$ and $I_{2}^{(3)}$, to the corresponding source nodes (i.e., to those with the signals $V_{3}^{(1)}$ and $V_{3}^{(3)}$, respectively) results in the simplified SFG shown in Fig. 9(c). This SFG-which corresponds to a Coates graph-comprises only source nodes. Applying Mason's rule, we obtain the three possible transmittances of the network:

$$
\begin{aligned}
& H_{1}=\frac{V_{3}^{(1)}}{V_{1}^{(1)}}=\frac{C_{1}}{C_{2}} z^{-3} \\
& H_{2}=\frac{V_{3}^{(2)}}{V_{1}^{(1)}}=0 \\
& H_{3}=\frac{V_{3}^{(3)}}{V_{1}^{(1)}}=\frac{C_{1}}{C_{2}} z^{-2} .
\end{aligned}
$$

\section{II. Analysis of General SC Networks}

In this section we generalize the method described in Section II by extending it to multiphase SC networks with arbitrary structure. Thus in addition to elements contained in SSN-type networks, general networks may contain:

\footnotetext{
${ }^{7}$ Note that from here onwards the subperiod $\tau$ has been normalized to unity. Thus the transmittances continue to relate voltage to current and not to charge.
}

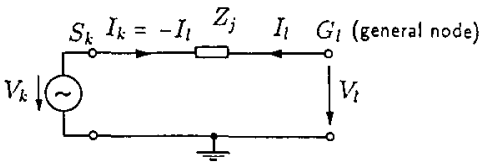

(a)
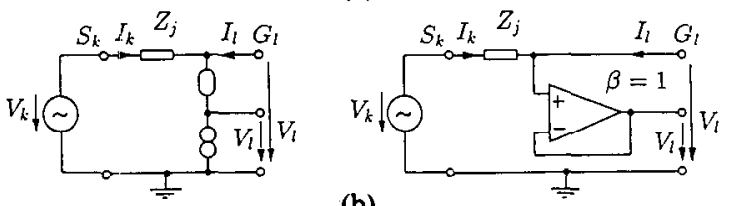

(b)

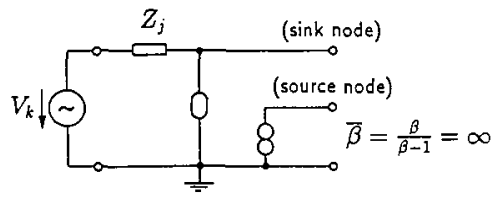

(c)
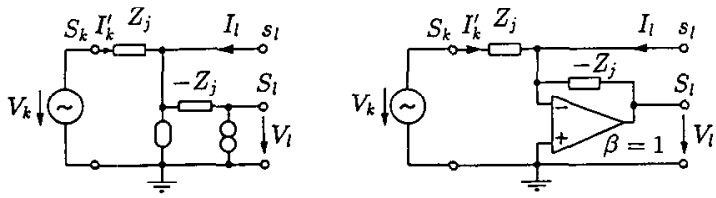

(d)

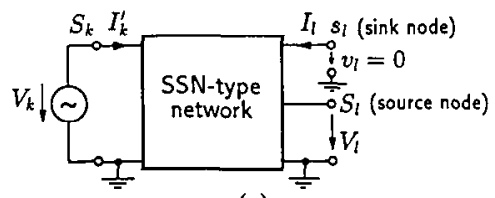

(e)

Fig. 10. SSN-network transformation. (a) Initial circuit. (b) Equivalent circuit with a dummy unity-gain amplifier. (c) Complementary transformed circuit. (d) Nullator-norator model of the final transformed circuit. (e) Final transformed circuit.

i) general nodes, i.e., nodes which are neither source nor sink nodes;

ii) differential input op amps;

iii) finite-gain amplifiers (including unity-gain buffers).

We shall now show that a general network can be converted into an equivalent SSN-type network by the so-called SSN-network transformation, which was introduced briefly in [17] and will be described in more detail below. Having carried out this transformation on a general network, the resulting equivalent SSN-type network can be treated as described in Section II.

\subsection{SSN-Network Transformation}

Consider the simple network shown in Fig. 10(a). It consists of an impedance $Z_{j}$ connected between a source node $S_{k}$ (with the node voltage $V_{k}$ ) and a general node $G_{l}$ (with the node voltage $V_{l}$ ). Our objective is to transform this network such that the general node $G_{l}$ is broken into two nodes: a source node $S$, with the same node voltage $V_{l}$ and a sink node $s_{l}$ supplying the same current $I_{l}$ to the transformed network (Fig. 10(e)). The transformed (SSNtype) network is equivalent to the original, even though the current leaving the source node $S_{k}$ (i.e., $I_{k}=-I_{l}$ in 


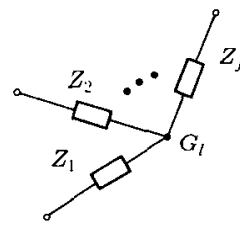

(a) $\stackrel{i}{\underline{2}}$

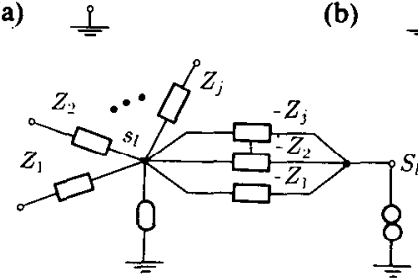

(c)

Fig. 11. Transformation of a general node $G_{i}$ by the SSN-network transformation. (a) Initial circuit. (b) Circuit with a dummy unity-gain amplifier. (c) Final circuit.

Fig. 10(a) and $I_{k}^{\prime}$ in Fig. 10(e)) may be different for the two networks (since $I_{k}$ and $I_{k}^{\prime}$ are supplied from source nodes, they need not be the same).

To transform the network of Fig. 10(a) into an SSN-type network we connect a series nullator-norator between the general node $G_{l}$ and ground (Fig. 10(b)). This does not affect the original circuit because a nullator and norator in series corresponds to an open circuit [21]. Nevertheless, it can be interpreted as the insertion of a unity-gain amplifier (gain $\beta=1$ ), as shown in Fig. 10(b). In order to obtain a source and sink node at $G_{l}$ we require a grounded norator and nullator. We can achieve this by applying the so-called complementary transformation [18], [19] to the network of Fig. 10(b), as shown in Fig. 10(c). Howcver, according to the complementary transform we now have the new gain $\bar{\beta}=\beta / \beta-1=\infty$ instead of the unity gain we started out with and now require. There is a second condition for the general node, however, that governs the current $I_{l}$, and that must also be satisfied, namely:

$$
I_{l}=\frac{V_{l}}{Z_{j}}-\frac{V_{k}}{Z_{j}}
$$

This is readily fulfilled by connecting an additional branch with the impedance $-Z_{j}$ between the new source (grounded norator) and sink (grounded nullator) node in Fig. 10(c), as shown in Fig. 10(d). This automatically restores our unity gain condition (see Fig. 10(d)) since

$$
\frac{V_{l}}{V_{k}}=-\frac{-Z_{j}}{Z_{j}}=1
$$

i.e., $\beta=1$; at the same time it completes the SSN-network transformation for the simple network of Fig. 10(a).

In what follows, it will be shown that the same procedure can be applied to any network incorporating general nodes. Consider, for example, branches with impedances $Z_{1}, Z_{2}, \cdots, Z_{j}$ incident to a general node $G_{l}$ as illustrated in Fig. 11(a). After the SSN-network transformation (Fig.

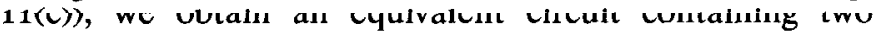

TABLE II

SSN-NETWORK OF A BRANCH WITH IMPEDANCE $Z$ InCident to at Least One General Node

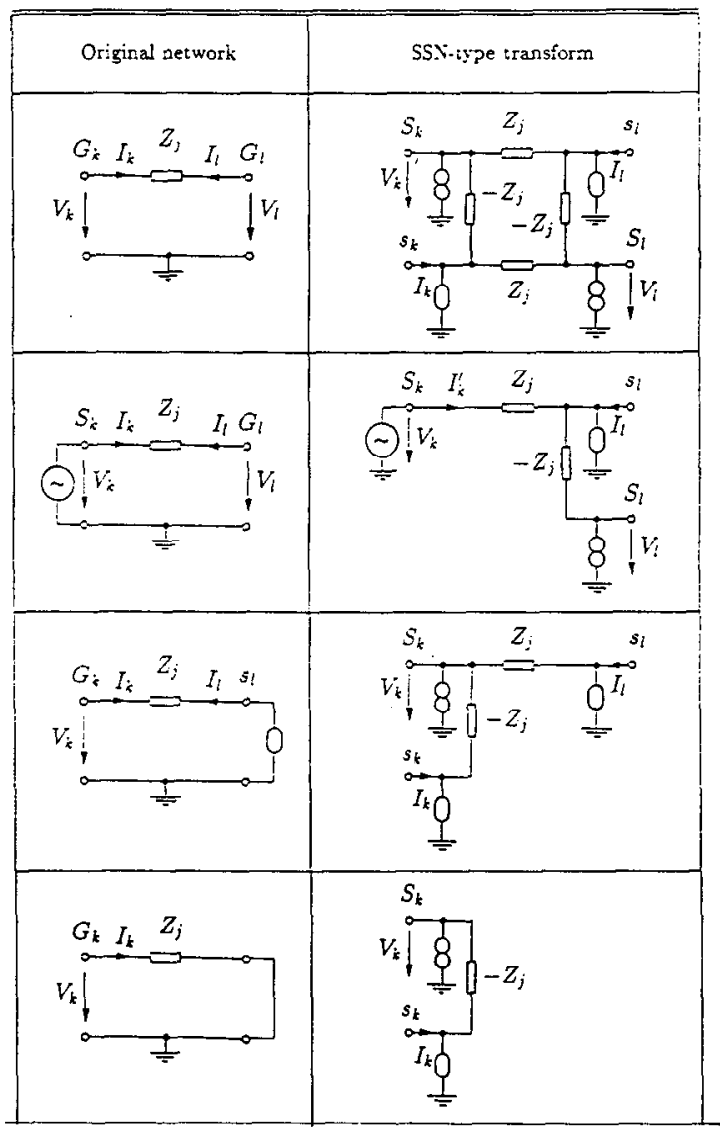

nodes: the source node $S_{l}$ and the sink node $s_{l}$, which replace the general node $G_{l}$ of the original circuit. This observation is formulated by the following proposition.

Proposition 4: Using the SSN-network transformation, any general node of an arbitrary network can be broken up into a source and sink node of an equivalent SSN-type network.

In Table II each possible configuration for a branch with an impedance $Z_{j}$ incident to at least one general node is shown, together with the corresponding SSN-type counterpart. We conclude from Table II that the impedance $Z_{j}$ can occur at most four times in the equivalent SSN-type network: twice with positive sign and twice with negative sign. This is shown in the first case in Table II in which $Z_{j}$ is connected between two general nodes $G_{k}$ and $G_{l}$. Note that the nullators and norators must be placed such that a nullator and a norator occurs at each end of an impedance $Z_{j}$. Thus for example, the two norators cannot be placed at the two upper nodes, and two nullators at the two lower nodes in the right-hand side of the top row of Table II.

The SSN-network transformation can readily be applied to any multiphase SC network. This is achieved by converting each SC branch of the original SC network

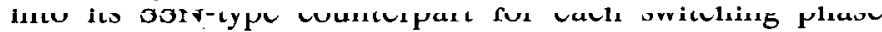




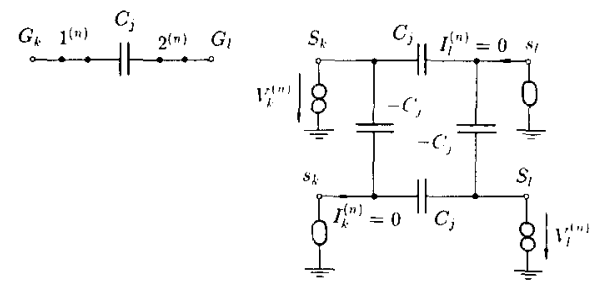

(a)

(b)

Fig. 12. SSN-network transformation of a capacitor $C_{j}$ connected in the $n$th phase between two general nodes $G_{k}$ and $G_{l}$. (a) Initial circuit configuration in the $n$th phase. (b) Transformed circuit configuration in the $n$th phase.

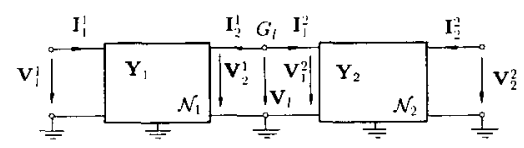

(a)

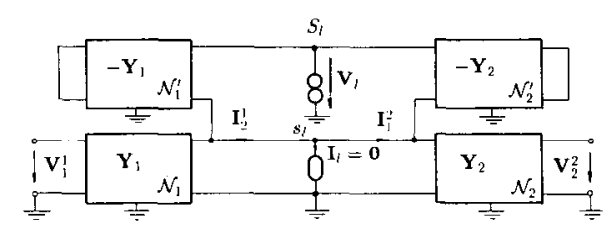

(b)

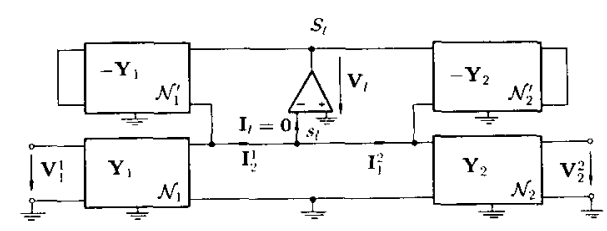

(c)

Fig. 13. Transformation of a cascade of two multiphase SSN-type SC two-ports $\mathscr{N}_{1}$ and $\mathscr{N}_{2}$. (a) Initial circuit. (b) Nullator-norator model of the SSN-type transformed circuit. (c) SSN-type transformed circuit.

according to Table II. For instance, a capacitor $C_{j}$, connected in the $n$th switching phase between two general nodes $G_{k}$ and $G_{l}$ (Fig. 12(a)), results in four capacitors (two positive and two negative) in the transformed SC network. During this phase, they are connected as shown in Fig. 12(b). The transform can also be applied to a cascade of two-ports. Consider, for example, a cascade of two SC $N$-phase two-ports $\mathscr{N}_{1}$ and $\mathscr{N}_{2}$ as shown in Fig. 13(a). We assume that each of the two-ports is an SSN-type network, but that the node $G_{l}$ in Fig. 13(a) is a general node. Each two-port can be described by its admittance matrix, i.e., $\boldsymbol{Y}_{1}$ and $\boldsymbol{Y}_{2}$ [15]. Note that voltages and currents in an SC $N$-phase network are $N$-dimensional vectors with elements corresponding to the individual switching phases (see expressions (B.2) and (B.3) in Appendix B) [15], [20]. The nullator-norator model of the transformed circuit is given in Fig. 13(b). Replacing the nullator-norator pair by an infinite-gain inverting op amp, we obtain the configuration in Fig. 13(c). The networks of Fig. 13(b) and (c) are equivalent to that in Fig. 13(a) in the sense that the voltage vector $V_{l}=V_{2}^{1}=V_{1}^{2}$ and the current vector $I_{2}^{1}=-I_{1}^{2}$ is the same for all these networks. An exact proof of this equivalence is given in

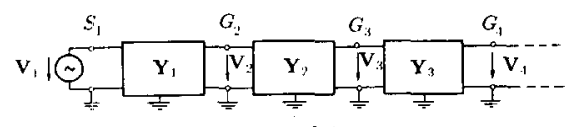

(a)

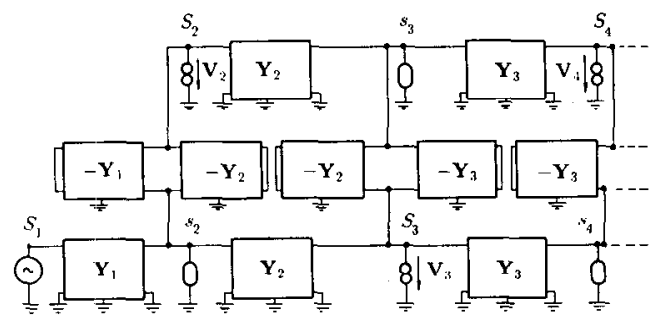

(b)

Fig. 14. SSN-network transformation of a cascade of arbitrarily many multiphase SSN-type SC two-ports. (a) Initial circuit. (b) Transformed circuit.

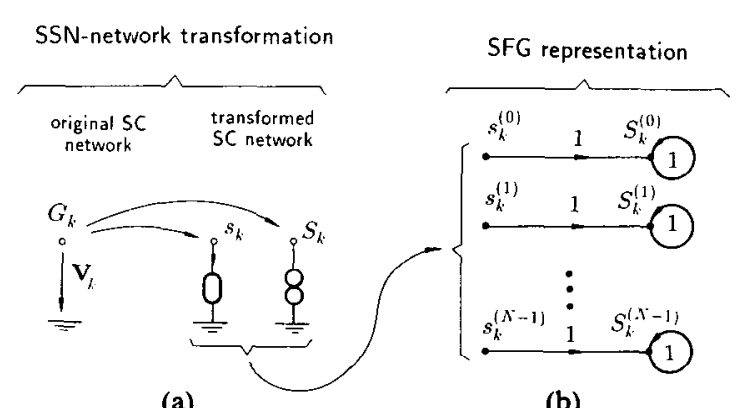

(a)

(b)

Fig. 15. Splitting of a general node $G_{k}$ to the corresponding SFG nodes. (a) SSN-network transformation. (b) SFG representation.

Appendix B. Consider now a cascade connection of arbitrarily many multiphase SSN-type SC two-ports (Fig. 14(a)). This network is converted by the SSN-network transformation into that shown in Fig. 14(b).

In the context of our SFG analysis method the concept of a transformed SSN-type SC network has primarily theoretical significance. The occurrence of negative components (e.g., capacitors) is, therefore, of no concern. In a forthcoming publication, we shall demonstrate how the SSN-network transformation can be used to convert a parasitic-sensitive circuit into a parasitic-insensitive one. There, the fact that negative components (i.e., capacitors) occur in the SSN-transformed network is also of no concern; using SFG node-scaling techniques, the sign of negative components can generally be reversed.

\subsection{Representation of Multiphase Switched Capacitors}

In our procedure, the SFG nodes represent the source nodes, sink nodes, and general nodes of the SC network, where the general nodes are split into source and sink nodes as illustrated in Fig. 15. Note that a general node, which is transformed into a source-sink node pair, is treated the same as an op amp (see Fig. 3). Whereas subgraphs corresponding to active elements are derived by the rules given in Section III-3.3 below, the branches modeling switched capacitors result from proposition 3 (or Table I). 


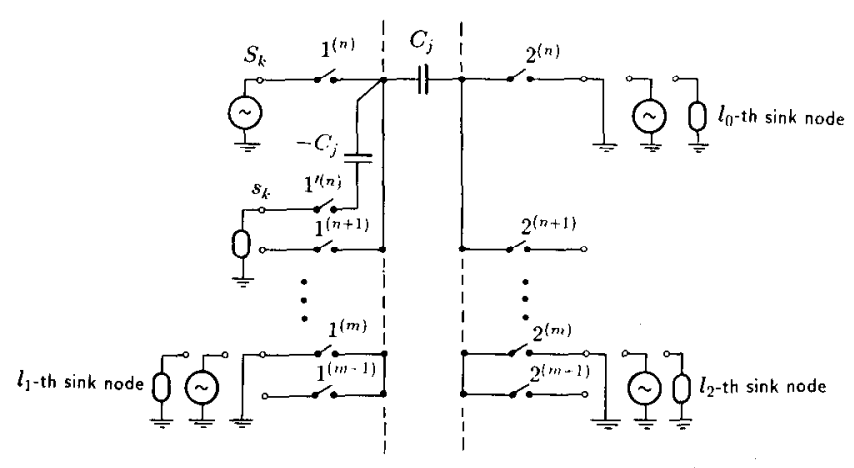

Fig. 16. A multiphase $\mathrm{SC} C_{j}$ of an arbitrary $\mathrm{SC}$ net-work.

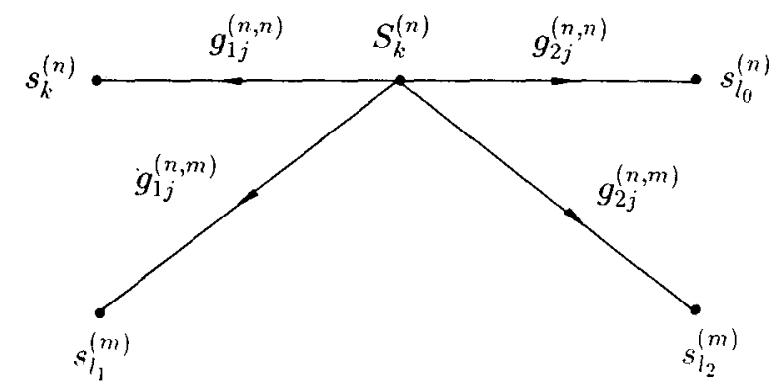

Fig. 17. Branches possibly generated in the SFG of an arbitrary SC network by a capacitor $C_{j}$ charged in the $n$th phase.

Assume that a capacitor $C_{j}$ of an arbitrary $N$-phase SC network is connected in the $n$th phase by at least one of its plates (e.g., by the left plate) to a general node, say to the node $G_{k}$ (Fig. 16). In order for any kind of charging or discharging to take place, the right capacitor plate may not be disconnected from the network in this phase. From Fig. 12 we conclude that in the transformed SSN-type SC circuit, apart from the capacitor $C_{j}$, also the capacitor $-C_{j}$ is charged to the voltage $V_{k}^{(n)}$ from the source node $S_{k}$. Indeed, in the $n$th switching phase, $-C_{j}$ is connected between the source node $S_{k}$ and the sink node $s_{k}$ (Fig. 16 ), and therefore generates a branch with transmittance $g_{1 j}^{(n, n)}$ in the SFG between these two nodes, as shown in Fig. 17. Since by definition the capacitor $-C_{j}$ has the negative charge of the capacitor $C_{j}$, the transmittance $g_{1 j}^{(n, n)}$ of this branch fulfills the following equation: ${ }^{8}$

$$
g_{1 j}^{(n, n)}=-g_{2 j}^{(n, n)}
$$

Note that $g_{2 j}^{(n, n)}$ is determined by expressions (1) or (2), depending on the existence of a discharging phase $\nu$.

The following two propositions can now be formulated. They permit the direct derivation of the SFG from a given general SC network without actually invoking the SSNnetwork transformation.

Proposition 5: Let $G_{k}$ be any general node of an arbitrary $N$-phase SC network. Then, assuming that $G_{k}$ is treated simultaneously as a source node $S_{k}$ and a sink node $s_{k}$ as illustrated in Fig. 15, transmittances of the SFG branches which start at the source node $S_{k}^{(n)}, n=$

\footnotetext{
${ }^{8}$ Here we assume, of course, that the other plate of $C_{j}$ is connected to a sink node in the $n$th phase and not to a source node or to ground, in
}

$0,1, \cdots, N-1$, and end in sink nodes other than $s_{k}^{(n)}$, as well as transmittances of those branches which start in source nodes other than $S_{k}^{(n)}$ and end in the sink node $s_{k}^{(n)}$, arc detcrmincd by proposition 3 (or equivalently by Table I). In addition, branches starting at the source node $S_{k}^{(n)}$ and ending in the sink node $s_{k}^{(n)}, n=0,1, \cdots, N-1$, must be accounted for. These branches correspond to capacitors connected by one of their plates to the node $G_{k}$ in the $n$th phase, and not disconnected from the network by the other plate in this phase. Let $C_{j}$ be one such capacitor. The transmittance $g_{1 j}^{(n, n)}$ between the nodes $S_{k}^{(n)}$ and $s_{k}^{(n)}$ that is generated by $C_{j}$ is given by (9).

This brings us to our final, sixth proposition.

Proposition 6: In the SFG of a general multiphase SC network, at most four branches, corresponding to a particular capacitor, can leave a source node (Fig. 17).

From proposition 5 it follows that in a general multiphase SC network, in addition to the three possible branches lcaving a source node in an SSN-type network, as postulated by proposition 1 (Fig. 8), a fourth branch with the transmittance $g_{1 j}^{(n, n)}$ may exist from the source node $S_{k}^{(n)}$ to the sink node $s_{k}^{(n)}$ corresponding to the general node $G_{k}$.

\subsection{Representation of Active Elements}

Although any general SC circuit node should, in principle, be modeled by the subgraph of Fig. 15(b), simplifications (analogous to Nathan's rules in matrix analysis [21], [22]) can be made, which correspond to constraints imposed by some of the active elements in certain switching phases (Table III). Note that the number of SFG sink and independent source nodes are both reduced by the same amount due to these simplifications, thus the possibility of node pairing, necessary to connect SFG sink nodes to source nodes by subgraphs of the type in Fig. 3(a), is maintained. Although, as shown in Appendix A, node pairing by any permutation is allowed, convenient pairing suggestions are illustrated in Table III.

The simplifications mentioned above are as follows.

i) The two voltages at the input nodes of a differential-input op amp will in general be equal (case 3 in Table III). Both nodes are combined into a single node whose voltage corresponds to the common input voltage. The currents must be modeled separately, however (i.e., by two different sink nodes). The fourth node, necessary for sink-source node pairing, corresponds to the op amp output node.

ii) The voltages of some nodes can be related to each other, e.g., by finite-gain amplification. In this case only the amplifier inputs are modeled as general nodes; the outputs are represented in the SFG by additional (dependent) source nodes. These are connected by direct gain branches (gain $A$ ) with the SFG source nodes corresponding to the amplifier inputs (cases 4 and 5 in Table III).

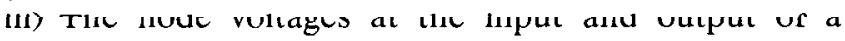


TABLE III

Representation of Grneral Nodes and Active Elements: Pairing Suggestions and Tyingi Ruif. FOR SFG NODES

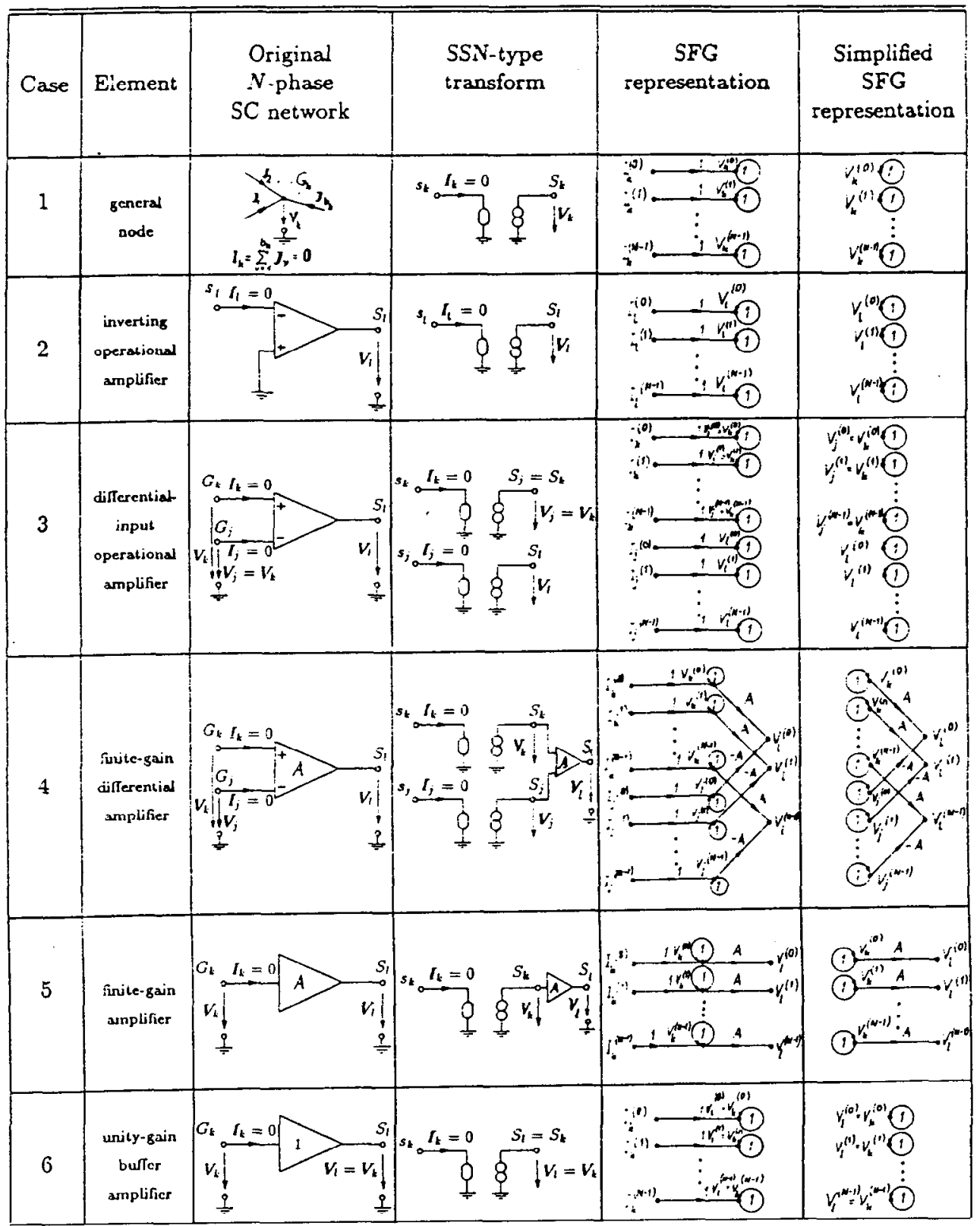

unity-gain buffer amplifier are equal; the two nodes are modeled by a single source node representing the common (input/output) voltage and by a singlc sink node corresponding to the buffer input current (case 6 in Table III).

\subsection{Further Illustrative Examples}

We shall now provide two illustrative examples that demonstrate our by-inspection procedure for the derivation of SFG's for general multiphase SC networks. In both examples the switching phases are numbered from 1 to $N$, as customary in the literature, and not from 0 to $N-1$, as was done, for convenience, in the theoretical presentation above.
Note that on the basis of proposition 5, general nodes are treated simultaneously as source and sink nodes. The method of dealing with them can be summarized as follows.

i) For any capacitor charged in a particular switching phase from a particular source (or general node), all other general nodes are treated as sink nodes; the corresponding SFG branch transmittances follow from the theory for SSN-type networks (Section II).

ii) Each capacitor charged in a particular switching phase $n$ from a particular general node $G_{k}$ generates an SFG branch starting at the source node $S_{k}^{(n)}$ and ending in the sink node $s_{k}^{(n)}$ associated with that general node. The transmittance of this branch is determined by proposition 5 (see also Table I). 
Example 2: Consider the 3-phase SC network [6] shown in Fig. 18(a). Note that the original single switch between capacitors $C_{1}$ and $C_{2}$ at node 2 has been split into two equivalent switches in series. This helps to visualize and localize the general node 2 (in practice this step may be left out). Beside the general node 2 , the circuit contains two source nodes, 1 and 4 , and one sink node, 3 . The general node $\mathbf{2}$ is connected to the circuit only in phase 2 and need be represented in the SFG only in this phase, namely by the sink node $I_{2}^{(2)}$ and the source node $V_{2}^{(2)}$. The corresponding SFG is shown in Fig. 18(b). Note that as a consequence of proposition 5 , nodes $V_{2}^{(2)}$ and $I_{2}^{(2)}$ are connected by branches with transmittances $-C_{1}$ and $-C_{2}\left(1-z^{-3}\right)$ (see Table I). The transmittance $-C_{1}$ follows from (1) and (9), since the capacitor $C_{1}$ is discharged in phase 1 . No such discharging phase exists for the capacitor $C_{2}$, and therefore, the respective transmittance, $-C_{2}\left(1-z^{-3}\right)$, is obtained from (2) and (9) with $N=3$. Fig. 18(c) shows the simplified SFG, i.e., that obtained after tying the sink nodes (representing the signals $I_{2}^{(2)}, I_{3}^{(1)}, I_{3}^{(2)}, I_{3}^{(3)}$ ) to the corresponding source nodes (i.e., to $V_{2}^{(2)}, V_{4}^{(1)}, V_{4}^{(2)}$, and $V_{4}^{(3)}$, respectively). Substituting $z^{3} \rightarrow z$, this SFG agrees with that published in [6]. Using Mason's formula we obtain any desired transfer quantity such as the voltage transfer function

$$
H_{43}=\frac{V_{k}^{(4)}}{V_{1}^{(3)}}=\frac{C_{2}}{C_{1}+C_{2}} z^{-5}=H_{21} .
$$

Thus for the overall transfer function $H$ we obtain

$$
H=\frac{V_{k}^{(2)}+V_{k}^{(4)}}{V_{1}^{(1)}+V_{1}^{(3)}}=\frac{C_{1}}{C_{1}+C_{2}} z^{-3}+\frac{C_{2}}{C_{1}+C_{2}} z^{-5}
$$

As expected, this equation describes an FIR filter with two taps. Clearly, extending the SC structure of Fig. 19(a) by further taps results in an FIR filter of a higher order.

\section{Conclusions}

A general by-inspection method for the closed-form analysis of arbitrary multiphase SC networks using signal-flow graph techniques has been presented. The derivation of the SFG is based on simple rules which are convenient not only for hand analysis but also for computer symbolic analysis and for the synthesis of SC networks. To obtain any desired transfer function, the wellknown Mason's formula can be used.

Although our procedure for the SFG derivation of a general SC network is direct, it can be interpreted as

$$
H_{22}=\frac{V_{4}^{(2)}}{V_{1}^{(2)}}=\frac{C_{1} C_{2}\left(z^{-3}-1\right) z^{-3}}{C_{2} C_{3} z^{-6}+\left[C_{1}\left(C_{1}-C_{3}\right)-2 C_{2} C_{3}\right] z^{-3}+C_{3}\left(C_{1}+C_{2}\right)}
$$

Example 3: Consider next the FIR SC filter proposed in [23]. The first two filter taps are shown in Fig. 19(a). In order to derive the SFG we note that the inputs of unity-gain buffer amplifiers 2,4 , and $k-1$ are general nodes and have the same node voltages as the output source nodes 3,5 , and $k$, respectively, i.e., $V_{2}^{(n)}=V_{3}^{(n)}$, $V_{4}^{(n)}=V_{5}^{(n)}$, and $V_{k-1}^{(n)}=V_{k}^{(n)}, n=1,2, \cdots, 4$. Because of these voltage equalities each node pair, i.e., $(2,3),(4,5)$, and $(k-1, k)$, can be represented by a single source node. Moreover, the first two pairs are connected to the network in phases 1 and 3 , and the last pair only in phases 2 and 4 . The SFG also contains the sink nodes $I_{2}^{(1)}, I_{2}^{(3)}, I_{4}^{(1)}, I_{4}^{(3)}, I_{k-1}^{(2)}$, and $I_{k-1}^{(4)}$ associated with the general network nodes 2,4 , and $\boldsymbol{k}-1$. The SFG and its simplified version are shown in Fig. 19(b) and (c), respectively. From the simplified SFG (Fig. 19(c)) we readily obtain the following transmittances:

$$
\begin{aligned}
& H_{21}=\frac{V_{k}^{(2)}}{V_{1}^{(1)}}=\frac{C_{2}}{C_{1}+C_{2}} z^{-5} \\
& H_{41}=\frac{V_{k}^{(4)}}{V_{1}^{(1)}}=\frac{C_{1}}{C_{1}+C_{2}} z^{3} \\
& I_{L 3} \quad \begin{array}{l}
V_{k}^{(2)} \\
V_{1}^{(3)}
\end{array} C_{1}+C_{2}{ }^{-3} X_{41}
\end{aligned}
$$

composed of two steps. In the first, a given SC network is replaced by a transformed one in which all critical nodes are either source or sink nodes (an SSN-type network). The second step permits the analysis of the network as an SSN-type network which allows straightforward use of the method presented in [1] and recapitulated in Section II. Due to the SSN-network transformation, SFG representations of different circuit elements (general nodes, active elements, etc.) are unified: the representation of inverting op amps, unity-gain buffers, and general nodes are identical (cf., Table III).

The same rules, which serve for the SFG derivation, can also be used to synthesize an SC network from a given SFG corresponding to a given transfer function. Freedom in pairing of SFG nodes (Appendix A) can be effectively exploited for the synthesis in order to obtain different SC realizations of the synthesized transfer function. Although from the mathematical point of view all possible pairing permutations are allowed, only some of them have an SC network interpretation. These are the ones in which pairs of SFG sink and source nodes corresponding to the same switching phase occur. The pairing possibility shown in an example of Appendix A is, therefore, not realizable.

The SSN-network transformation introduced in [17] and elaborated on in this paper is also useful for direct network synthesis. By this transformation, SSN-type (or even strav-insensitive) SC realizations of a given transfer function can be obtained on the basis of an arbitrary 


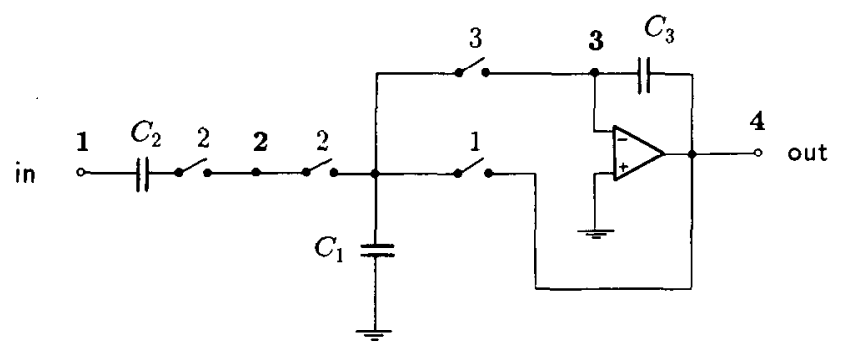

(a)

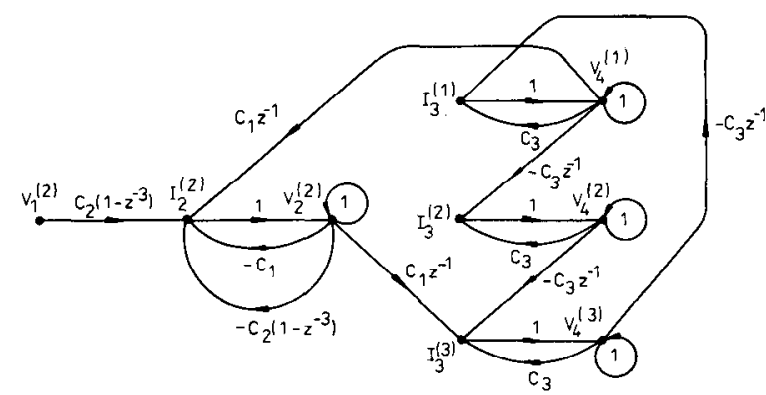

(b)

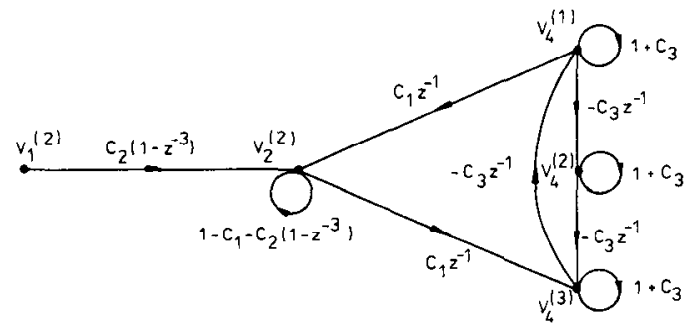

(c)

Fig. 18. SC circuit analyzed in [6]. (a) 3-phase SC circuit. (b) Corresponding SFG. (c) Simplified SFG.

realization. More on this feature of the transformation will be published shortly.

An additional interesting feature of our SFG derivation is that for a general SC network (i.e., with general nodes) there are at most four branches corresponding to a given capacitor; for an SSN-type SC network there are at most three branches; and for a stray-insensitive SC network, only two branches starting at a particular SFG source node (cf., propositions, 1, 2, and 6). This observation simplifies both circuit analysis and synthesis.

\section{APPENDIX A}

Generally, an arbitrary $N$-phase SC network may be completely described by Kirchhoff's voltage law (KVL) and current (or charge) law (KCL). The corresponding equations are thereby written separately for each switching phase. KVL may be implemented simply by choosing node voltages of all critical nodes as primary variables. In order to fulfil KCL for any node, say for the $k$ th node, we define the signal $\boldsymbol{I}_{k}=\mathbf{0}$ as shown in the first row of Table III. The $k$ th node is then described by the signals $V_{k}$ and $\boldsymbol{I}_{k}=\mathbf{0}$ which arc split in the corresponding SFG into the signals $V_{k}^{(0)}, V_{k}^{(1)}, \cdots, V_{k}^{(N-1)}$ and

$$
I_{k}^{(0)}, I_{k}^{(1)}, \cdots, I_{k}^{(N-1)}=0
$$

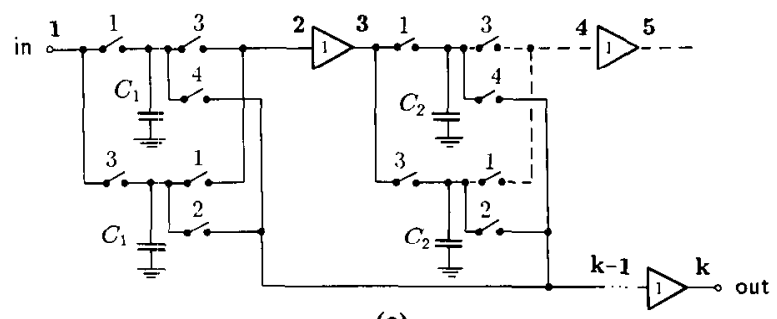

(a)
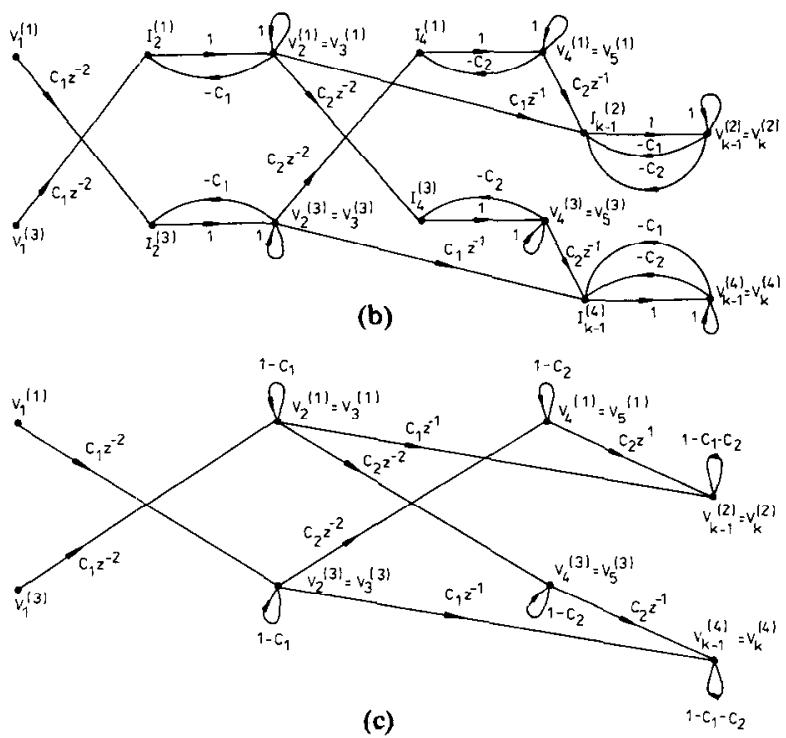

Fig. 19. FIR SC filter described in [23]. (a) FIR SC filter cut to two taps. (b) Corresponding SFG. (c) Simplified SFG.

where the superscripts in parentheses correspond to individual switching phases.

Some nodes may be at virtual ground. These so-called sink nodes are described only by their currents. Some other nodes (the source nodes) are described only by their node voltages. General nodes, i.e., those described by both their node voltages and their currents, can then be interpreted as source and sink nodes simultaneously (cf., a concept of the SSN-type equivalent SC network presented in Section III-3.1). In fact, in general, the following situations may occur:

i) one or more source nodes are input nodes to the system;

ii) some source nodes can be at the outputs of finitegain amplifiers; thus their node voltages depend directly on node voltages of other nodes, namely, of those at the amplifier inputs;

iii) the node voltages of the rest of the nodes follow from the equilibrium in the network such that (A.1) is fulfilled.

The source nodes belonging to the first two groups are called the dependent source nodes, while those from the third group are called independent source nodes. Source nodes associated with general nodes are clearly independent in the above sense. Active elements reduce the number of sink nodes and the number of independent source nodes but, as shown in Table III, both these numbers always remain equal. 


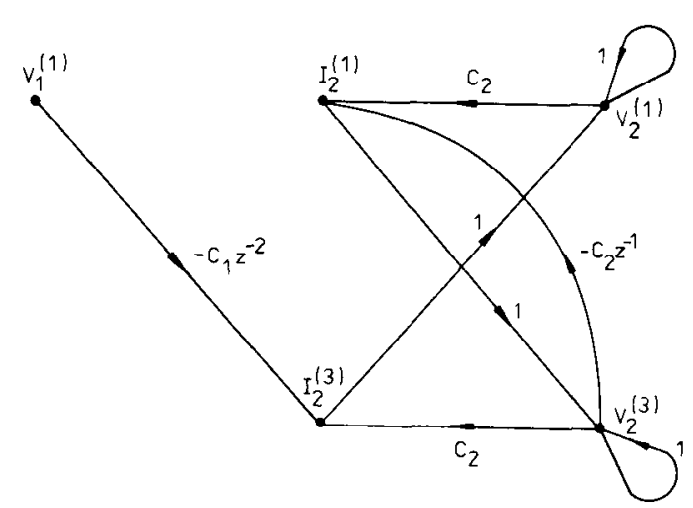

Fig. 20. Alternative SFG for the SC delay element of Fig. 9(a).

In order to represent sink nodes in the SFG, (A.1) must be fulfilled for all sink nodes in all switching phases. This has been done by pairing sink nodes with source nodes and by connecting each pair with a subgraph of the type shown in Fig. 3(a). Clearly, if we exclude ill-conditioned SC networks (i.e., those in which some source nodes can have arbitrary node voltages), all possible pairing permutations are allowed. Those given in Table III should, therefore, be understood merely as useful suggestions.

Example: Another pairing possibility for the nodes of the SFG in Fig. 9(b) is illustrated in Fig. 20. Naturally, we obtain, for this new SFG, the same transmittances as those given in Example 1. Note, however, that this pairing would not lead to a physically realizable network because the source and sink nodes representing an op amp are interconnected in different phases.

\section{Appendix B}

In this appendix, we prove that the $N$-phase SC networks of Fig. 13(b) and (c) are equivalent to that in Fig. 13(a) in the sense that the signals $V_{j}=V_{2}^{1}=V_{1}^{2}$ and $I_{2}^{1}=$ $-I_{1}^{2}$ are modeled accurately between all these networks. To this end we use the four-port matrix theory introduced in [20].

Assume that each of the two-ports $\mathscr{N}_{1}$ and $\mathscr{N}_{2}$ is an SSN-type network, but that the node $G_{l}$ in Fig. 13(a) is a general node. Each two-port can be described by its admittance matrix, i.e., $Y_{1}$ and $Y_{2}$. Thus we have

$$
\left[\begin{array}{c}
\boldsymbol{I}_{1}^{\xi} \\
\boldsymbol{I}_{2}^{\xi}
\end{array}\right]=\boldsymbol{Y}_{\xi}\left[\begin{array}{c}
\boldsymbol{V}_{1}^{\xi} \\
\boldsymbol{V}_{2}^{\xi}
\end{array}\right], \quad \xi=1,2
$$

where $\xi$ refers to the network $\mathscr{N}_{1}$ and $\mathscr{N}_{2}$;

$$
\begin{gathered}
\boldsymbol{I}_{\zeta}^{\xi}=\left[I_{\zeta}^{\xi(0)}, I_{\zeta}^{\xi(1)}, \cdots, I_{\zeta}^{\xi(N-1)}\right]^{T} \\
\boldsymbol{V}_{\eta}^{\xi}=\left[V_{\eta}^{\xi(0)}, V_{\eta}^{\xi(1)}, \cdots, V_{\eta}^{\xi(N-1)}\right]^{T}, \\
\zeta, \eta=1,2
\end{gathered}
$$

and

$$
Y_{\xi}=\left[\begin{array}{ll}
{\left[y_{11}^{\xi}\right]} & {\left[y_{12}^{\xi}\right]} \\
{\left[y_{21}^{\xi}\right]} & {\left[y_{22}^{\xi}\right]}
\end{array}\right] .
$$

Note that $\zeta, \eta=1$ refers to the two-port input and $\zeta$,

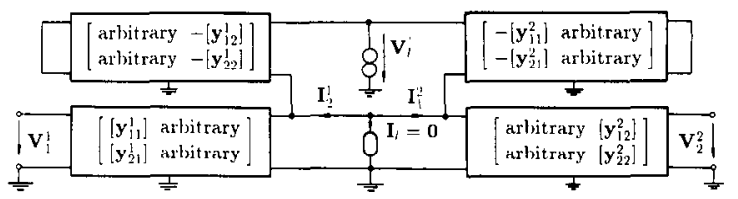

(a)

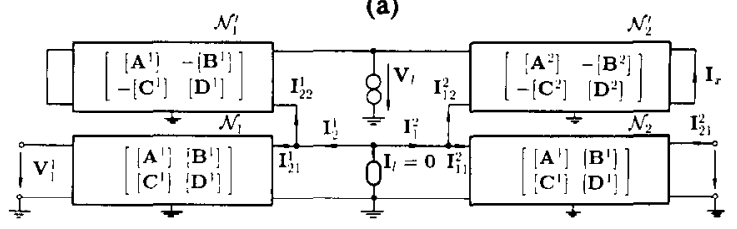

(b)

Fig. 21. SSN-network transformation of a cascade of two multiphase SSN-type SC two-ports $\mathscr{N}_{1}$ and $\mathscr{N}_{2}$. (a) Transformed circuit. (b) Nullator-norator model of the SSN-type transformed circuit.

$\eta=2$ to the output. The submatrices in (B.4) are given by

$$
\left[\boldsymbol{y}_{\zeta \eta}^{\xi}\right]=\left[\begin{array}{cccc}
y_{\xi \eta}^{\xi(0,0)} & y_{\xi \eta}^{\xi(0,1)} & \cdots & y_{\xi \eta}^{\xi(0, N-1)} \\
y_{\zeta \eta}^{\xi(1,0)} & y_{\xi \eta}^{\xi(1,1)} & \cdots & y_{\xi \eta}^{\xi(1, N-1)} \\
\vdots & \vdots & \ddots & \vdots \\
y_{\xi \eta}^{\xi(N-1,0)} & y_{\xi \eta}^{\xi(N-1,1)} & \cdots & y_{\xi \eta}^{\xi(N-1, N-1)}
\end{array}\right]
$$

Currents $I_{\xi}^{\xi(m)}$, voltages $V_{\eta}^{\xi(n)}$, and admittances $y_{\xi \eta}^{\xi(m, n)}$ correspond to the $m$ th and /or to the $n$th switching phase.

Notice that (B.1) can also be written in the form:

$$
\begin{aligned}
& {\left[\begin{array}{c}
\boldsymbol{I}_{1}^{\xi} \\
\boldsymbol{I}_{2}^{\xi}
\end{array}\right]=\left[\begin{array}{ll}
{\left[\boldsymbol{y}_{11}^{\xi}\right]} & \text { arbitrary } \\
{\left[\boldsymbol{y}_{21}^{\xi}\right]} & \text { arbitrary }
\end{array}\right]\left[\begin{array}{c}
\boldsymbol{V}_{1}^{\xi} \\
\mathbf{0}
\end{array}\right]} \\
& +\left[\begin{array}{ll}
\text { arbitrary } & {\left[y_{12}^{\xi}\right]} \\
\text { arbitrary } & {\left[\boldsymbol{y}_{22}^{\xi}\right]}
\end{array}\right]\left[\begin{array}{c}
\mathbf{0} \\
\boldsymbol{V}_{2}^{\xi}
\end{array}\right] . \\
& \xi=1,2 .
\end{aligned}
$$

Equation (B.6) can be used to convert the SC network of Fig. 13(a) into that shown in Fig. 21(a). With a little thought it is clear that the SSN-network transformation of the network in Fig. 13(a) results in a particular version of the network in Fig. 21(a), namely that shown in Fig. 13(c) (its nullator-norator model is given in Fig. 13b and Fig. 21(b)).

Describe now the two-ports $\mathscr{N}_{1}$ and $\mathscr{N}_{2}$ by their chain matrices:

$$
\left[\begin{array}{ll}
{\left[A^{1}\right]} & {\left[B^{1}\right]} \\
{\left[C^{1}\right]} & {\left[D^{1}\right]}
\end{array}\right] \text { and }\left[\begin{array}{ll}
{\left[A^{2}\right]} & {\left[B^{2}\right]} \\
{\left[C^{2}\right]} & {\left[D^{2}\right]}
\end{array}\right]
$$

respectively. From Fig. 21(b) the following expressions can be derived:

for network $\mathscr{N}_{1}^{\prime}:\left[A^{1}\right] V_{l}-\left[B^{1}\right] I_{22}^{1}=0$

for network $\mathscr{N}_{1}: \quad V_{1}^{1}=-\left[B^{1}\right] I_{21}^{1}$

for network. $\mathscr{N}_{2}^{\prime}: \quad V_{l}=\left[B^{2}\right] I_{x}$

$$
I_{12}^{2}=\left[D^{2}\right] I_{x}
$$


and for network $\mathscr{N}_{2}$ :

$$
\begin{aligned}
& {\left[A^{2}\right] V_{2}^{2}-\left[B^{2}\right] I_{21}^{2}=0} \\
& I_{11}^{2}=\left[C^{2}\right] V_{2}^{2}-\left[D^{2}\right] I_{21}^{2} .
\end{aligned}
$$

From (B.11) and (B.12) we obtain

$$
I_{11}^{2}=\left(\left[C^{2}\right]-\left[D^{2}\right]\left[B^{2}\right]^{-1}\left[A^{2}\right]\right) V_{2}^{2} .
$$

Moreover, from (B.9) and (B.10) we have

$$
I_{12}^{2}=\left[D^{2}\right]\left[B^{2}\right]^{-1} V_{l}
$$

Thus from (B.7) and (B.14) we obtain

$$
\left[A^{1}\right]^{-1}\left[B^{1}\right] I_{22}^{1}=\left[B^{2}\right]\left[D^{2}\right]^{-1} I_{12}^{2} .
$$

Using equality $I_{2}^{1}=-I_{1}^{2}$ we obtain from (B.8) and (B.13)

$$
I_{22}^{1}-\left[B^{1}\right]^{-1} V_{1}^{1}=\left(\left[D^{2}\right]\left[B^{2}\right]^{-1}\left[A^{2}\right]-\left[C^{2}\right]\right) V_{2}^{2}-I_{12}^{2} \text {. }
$$

Now from (B.15) and (B.16) we compute

$$
\begin{aligned}
I_{22}^{1} & =\left(\left[D^{2}\right]\left[B^{2}\right]^{-1}\left[A^{1}\right]^{-1}\left[B^{1}\right]+1\right)^{-1} \\
& \cdot\left\{\left[B^{1}\right]^{-1} V_{1}^{1}+\left(\left[D^{2}\right]\left[B^{2}\right]^{-1}\left[A^{2}\right]-\left[C^{2}\right]\right)\right\} V_{2}^{2} .
\end{aligned}
$$

Finally, from (B.8) and (B.17) we obtain

$$
\begin{aligned}
I_{1}^{2}= & -I_{2}^{1}=-I_{21}^{1}-I_{22}^{1} \\
= & \left(\left[A^{1}\right]^{-1}\left[B^{1}\right]+\left[B^{2}\right]\left[D^{2}\right]^{-1}\right)^{-1} \\
& \cdot\left\{\left[A^{1}\right]^{-1} V_{1}^{1}+\left(\left[B^{2}\right]\left[D^{2}\right]^{-1}\left[C^{2}\right]-\left[A^{2}\right]\right) V_{2}^{2}\right\},
\end{aligned}
$$

Similarly, we can show that

$$
\begin{aligned}
V_{l} & =\left(\left[B^{1}\right]^{-1}\left[A^{1}\right]+\left[D^{2}\right]\left[B^{2}\right]^{-1}\right)^{-1} \\
& \cdot\left\{\left[B^{1}\right]^{-1} V_{1}^{1}+\left(\left[D^{2}\right]\left[B^{2}\right]^{-1}\left[A^{2}\right]-\left[C^{2}\right]\right) V_{2}^{2}\right\} .
\end{aligned}
$$

On the other hand, from the circuit of Fig. 13(a) we can immediately write

for network $\mathscr{N}_{1}: V_{1}^{1}=\left[A^{1}\right] V_{l}+\left[B^{1}\right] I_{1}^{2}$

and for network $\mathscr{N}_{2}$ :

$$
\begin{aligned}
V_{l} & =\left[A^{2}\right] V_{2}^{2}-\left[B^{2}\right] I_{2}^{2} \\
I_{1}^{2} & =\left[C^{2}\right] V_{2}^{2}-\left[D^{2}\right] I_{2}^{2} .
\end{aligned}
$$

From (B.21) and (B.22) we obtain

$$
\left[B^{2}\right]^{-1} V_{l}-\left[D^{2}\right]^{-1} I_{1}^{2}=\left(\left[B^{2}\right]^{-1}\left[A^{2}\right]-\left[D^{2}\right]^{-1}\left[C^{2}\right]\right) V_{2}^{2} \text {. }
$$

Equations (B.20) and (B.23) can be transformed into the following expressions

$$
\begin{aligned}
& {\left[B^{1}\right]^{-1}\left[A^{1}\right] V_{l}+I_{1}^{2}=\left[B^{1}\right]^{-1} V_{1}^{1}} \\
& {\left[D^{2}\right]\left[B^{2}\right]^{-1} V_{l}-I_{1}^{2}=\left(\left[D^{2}\right]\left[B^{2}\right]^{-1}\left[A^{2}\right]-\left[C^{2}\right]\right) V_{2}^{2}}
\end{aligned}
$$

or

$$
\begin{aligned}
V_{l}+\left[A^{1}\right]^{-1}\left[B^{1}\right] I_{1}^{2} & =\left[A^{1}\right]^{-1} V_{1}^{1} \\
-V_{l}+\left[B^{2}\right]\left[D^{2}\right]^{-1} I_{1}^{2} & =\left(\left[B^{2}\right]\left[D^{2}\right]^{-1}\left[C^{2}\right]-\left[A^{2}\right]\right) V_{2}^{2} .
\end{aligned}
$$

From (B.24) and (B.25) we again obtain (B.19), and similarly, from (B.26) and (B.27) we obtain (B.18). Hence, we have proven that the signals $V_{l}$ and $I_{1}^{2}$ in Fig. 13(a) are equal to $V_{l}$ and $I_{1}^{2}$, respectively, in Fig. 13(b) and (c).

\section{REFERENCES}

[1] A. Dabrowski and G. S. Moschytz, "Direct by-inspection derivation of signal-flow graphs for multiphase stray-insensitive switched-capacitor networks," vol. 25, pp. 387-389, Mar. 1989 Electron. Lett.

[2] G. S. Moschytz and J. J. Mulawka, "Direct analysis of stray-insensitive switched-capacitor networks using signal flow graphs," Inst. Elect. Eng. Proc., part G, vol. 133, pp. 145-153, June 1986.

[3] G. S. Moschytz and U. W. Brugger, "Signal-flow graph analysis of SC nctworks," Inst. Elect. Eng. Proc., part G, vol. 131, pp. 72-85, Apr. 1984.

[4] R. Unbehauen and A. Cichocki, MOS Switched-Capacitor and Continuous Time Integrated Circuits and Systems. Berlin, Germany: Springer Verlag, 1989.

[5] S. D. Bedrosian and S. Refai, "A flow graph transformation technique specifically for discrete time networks," $J$. Franklin Inst., vol. 315, pp. 27-36, Jan. 1983.

[6] __ "Switched-capacitor networks: Analysis and fault diagnosis," in Proc. 1982 IEEE Int. Large Scale Systems Symp., Virginia Beach, VA, pp. 316-320, Oct. 1982.

[7] M. Bon and A. Kończykowska, "All-symbolic analysis techniques for multiphase switched capacitor networks," in Proc. Fifth European Conf. Circuit Theory Design, The Hague, The Netherlands, pp. 655-660, Aug. 1981.

[8] X.-F. Zhou and Z.-G. Shen, "Signal flow graph analysis of switched-capacitor networks," Int. J. Circuit Theory Appl., vol. 13, pp. $179-189$, Apr. 1985 .

[9] R. Dostal and J. Mikula, "Analysis and synthesis of switched capacitor networks using flow graphs," in Proc. Seventh European Conf. Circuit Theory Design, Prague, Czechoslovakia, pp. 705-708, Sept. 1985.

[10] J. J. Mulawka and G. S. Moschytz, "A by-inspection analysis of SC networks using direct topological rules," Inst. Elect. Eng. Proc., part G, vol. 132, pp. 255-265, Dec. 1985.

[11] S. J. Harrold, I A W. Vance, and D. G. Haigh, "Second-order switched-capacitor bandpass filter implemented in GaAs," Electron. Lett., vol. 21, pp. 494-496, May 1985.

[12] J. A. Phillips, S. J. Harrold, and D. G. Haigh, "A GaAs switchedcapacitor bandpass filter operating at a frequency of $250 \mathrm{MHz}$ " in Proc. IEE Coll. IC's above $1 \mathrm{GHz}-$ Fabrication Circuit Design, London, Great Britain, pp. 15/1-15/5, Mar. 1986.

[13] C. Toumazou and D. G. Haigh, "Design of a high-gain, single-stage operational amplifier for GaAs switched-capacitor filters," Electron. Lett., vol. 23, pp. 752-754, July 1987.

[14] M. Hasler, "Stray capacitance insensitive switched capacitor filters," in Proc. IEEE Int. Symp. Circuits Syst., pp. 42-45, 1981.

[15] E. Hökenek and G. S. Moschytz, "Analysis of multiphase switched-capacitor (m.s.c.) networks using the indefinite admittance matrix (i.a.m.)," Proc. Inst. Elect. Eng., part G, vol. 127, pp. 226-241, Oct. 1980.

[16] T. Enomoto, T. Ishihara, and M.-A. Yasumoto, "Integrated tapped MOS analogue delay line using switched capacitor technique," Electron. Lett., vol. 18, pp. 193-194, Mar. 1982.

[17] A. Dabrowski and G. S. Moschytz, "Source-sink-node(SSN)-network transformation," IEEE Trans. Circuits Syst., vol. 37, pp. $656-658$, this issue.

[18] N. Fliege "Complementary transformation of feedback systems," IEEE Trans. Circuit Theory, vol. CT-20, pp. 137-139, Mar. 1973.

[19] G. S. Moschytz and P. Horn, "Optimising two commonly used active-filter building blocks using the complementary transformation," Inst. Elec. Eng. J. Electron. Circuits Syst., vol. 1, pp. 125-132, July 1977.

[20] G. S. Moschytz, "Elements of four-port matrix theory as required for SC network analysis," Int. J. Circuit Theory Appl., vol. 15, pp. 235-249, 1987.

[21] G. S. Moschytz, Linear Integrated Networks: Fundamentals. New York: Van Nostrand Reinhold, 1974.

[22] A. Nathan, "Matrix analysis of networks having infinite-gain operational amplifiers," Proc, IRE, vol. 49, pp. 1577-1578, Oct. 1961.

[23] D. C. von Grünigen, "Entwurfs- und Analyseverfahren für Filter mit geschalteten Kondensatoren," Ph.D. dissertation, Diss. ETH No. 7380, Swiss Federal Institute of Technology, Zürich, 1983. 


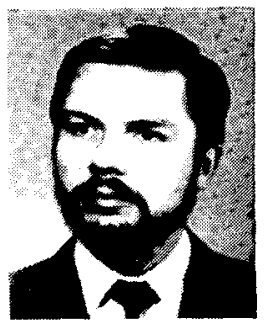

Adam Dagbrowski (M'87) received the M.S. degree in electrical engineering from the Technical University of Poznań, Poland, in 1976, the M.S. degree in mathematics from Adam Mickiewicz. University, Poznań, in 1977, and the Ph.D. degree in electronics from the Technical University of Poznań in 1983.

Since 1976 he has been with the Institute of Electronics and Telecommunication at the Technical University of Poznań, where he is now Associate Professor. He has visited Technische Universität in Wien, Austria, during 1981-1982. In the period 1984-1986 he was on leave at Ruhr-Universität, Bochum, Germany, as an Alexander von Humboldt Fellow. He has also visited Eidgenössische Technische Hochschule, Zürich, Switzerland, during the academic year 1987-1988. His research interests include general network theory, digital signal processing, and sampled-data filter synthesis.

Dr. Dąbrowski is a member of the Polish Society of Theoretical and Applied Electrical Sciences and the Association of Polish Electrical Engineers.

\section{里}

George S. Moschytz (M'65-SM'77-F'78) received the M.S. and Ph.D. degrees in electrical engineering from the Swiss Federal Institute of Technology, Zurich, Switzerland, in 1958 and 1962, respectively.

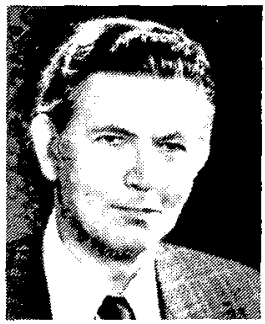

From 1960 to 1962 he was with the RCA Laboratories Ltd., Zurich, Switzerland. In 1963 he joined Bell Telephone Laboratories, Inc., Holmdel, NJ. Since 1973 he has been Professor of Electrical Engineering and Director of the Institute for Signal and Information Processing at the Swiss Federal Institute of Technology. He is author of Linear Integrated Networks: Fundamentals (New York: Van Nostrand Reinhold, 1974), Linear Integrated Networks: Design (New York, Van Nostrand Reinhold, 1975), co-author of the Active Filter Design Handbook (London: Wiley, 1981), and editor of MOS Switched-Capacitor Filters: Analysis and Design (New York: IEEE Press, 1984). He has authored numerous papers in the field of network theory, design, and sensitivity, and holds several patents in these areas. His present interests are in the fields of digital, switchedcapacitor, and adaptive filters, neural networks for signal processing, and the application of signal processing techniques to biological systems.

Dr. Moschytz is president of the IECE Swiss Chapter on Digital Communication Systems and a member of the Swiss Electrotechnical Society. From 1981 to 1982 he was President of the Swiss Section of the IEEE. He has held several terms in the Adcom of the IEEE Circuits and Systems Society as well as on the Editorial Board of the Proceedings of the IEEE and has been associate editor of the IEEE Circuits and Systems Magazine. He is also an associate editor of several other technical journals. 